\title{
Polymer Brushes on Graphitic Carbon Nitride for Patterning and as a SERS Active Sensing Layer via Incorporated Nanoparticles
}

\author{
Wenbo Sheng, Wei Li, Deming Tan, Panpan Zhang, En Zhang, Evgeniya Sheremet, \\ Bernhard V.K.J. Schmidt, Xinliang Feng, Raul D. Rodriguez, * Rainer Jordan,* and Ihsan Amin*
}

Cite This: ACS Appl. Mater. Interfaces 2020, 12, 9797-9805

Read Online

\section{ACCESS \\ Џlll Metrics \& More \\ Article Recommendations \\ Supporting Information}

ABSTRACT: Graphitic carbon nitride ( $\mathrm{gCN}$ ) has a broad range of promising applications, from energy harvesting and storage to sensing. However, most of the applications are still restricted due to gCN poor dispersibility and limited functional groups. Herein, a direct photografting of gCN using various polymer brushes with tailorable functionalities via UV photopolymerization at ambient conditions is demonstrated. The systematic study of polymer brush-functionalized gCN reveals that the polymerization did not alter the inherent structure of gCN. Compared to the pristine gCN, the gCN-polymer composites show good dispersibility in various solvents such as water, ethanol, and tetrahydrofuran (THF). Patterned polymer brushes on gCN can be realized by employing

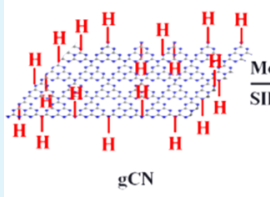
$\mathrm{CN}$

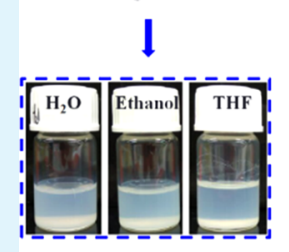

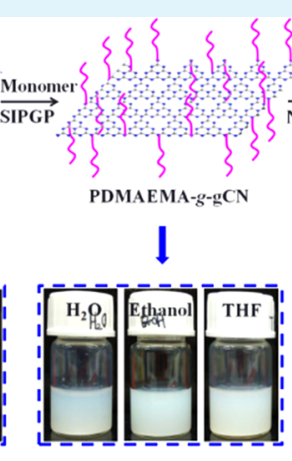

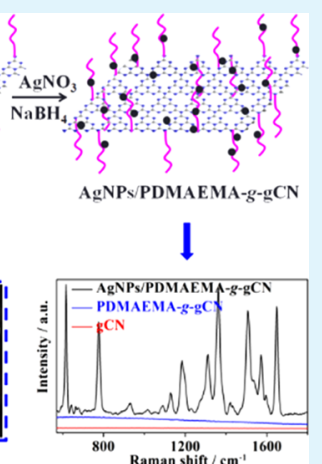
photomask and microcontact printing technology. The polymer brushes with incorporated silver nanoparticles (AgNPs) on gCN can act as a multifunctional recyclable active sensing layer for surface-enhanced Raman spectroscopy (SERS) detection and photocatalysis. This multifunctionality is shown in consecutive cycles of SERS and photocatalytic degradation processes that can be applied to in situ monitor pollutants, such as dyes or pharmaceutical waste, with high chemical sensitivity as well as to water remediation. This dual functionality provides a significant advantage to our AgNPs/polymer-gCN with regard to state-of-the-art systems reported so far that only allow SERS pollutant detection but not their decomposition. These results may provide a new methodology for the covalent functionalization of gCN and may enable new applications in the field of catalysis, biosensors, and, most interestingly, environmental remediation.

KEYWORDS: graphitic carbon nitride, polymer brushes, microcontact printing, surface-enhanced Raman spectroscopy, photocatalysis

\section{INTRODUCTION}

Graphitic carbon nitride $\left(\mathrm{gC}_{3} \mathrm{~N}_{4}\right.$ or $\left.\mathrm{gCN}\right)$, as a metal-free conjugated two-dimensional polymer, has attracted broad interdisciplinary attention in areas such as catalysis, ${ }^{1-6}$ solar energy conversion, ${ }^{7-9}$ environmental remediation, ${ }^{10-12}$ actuator, ${ }^{13}$ and so on. ${ }^{14}$ However, gCN has poor dispersibility in water and most organic solvents, which is one of the most critical limitations to realize its potential applications, in particular with large-scale fabrication using a solutionprocessable technology. Dispersibility can be improved by exfoliating gCN into single- or few-layer nanosheets with ultrasonication for more than $10 \mathrm{~h} .{ }^{15,16}$ The known methods for the functionalization of $\mathrm{gCN}$ not only require multistep processes but also destroy the intrinsic structure and compromise the gCN excellent properties. These issues can be partly overcome by grafting small molecules. ${ }^{17,18}$ Nevertheless, grafting small molecules does not necessarily introduce improved processing, which is of significant importance for film fabrication and device design. ${ }^{19,20}$ It is, therefore, highly desirable to develop new methods to introduce functionalities while improving the dispersibility and processability of gCN without destroying its original structure.

It is well known that primary and secondary amine functional groups are generated in gCN because of incomplete precursor condensation. $^{21}$ These amine functional groups are important for surface functionalization, especially to graft polymer brushes via surface-initiated photografting and photopolymerization (SIPGP). Recently, SIPGP was successfully used for the fabrication of polymer brushes on a wide range of substrates, including hexagonal boron nitride, ${ }^{22}$ diamond, ${ }^{23}$ graphene, ${ }^{24}$ carbon nanomembranes, ${ }^{25,26}$ carbon nanotubes, ${ }^{27}$ polydopamine, ${ }^{28-30}$ and so on. In comparison with other grafting techniques, SIPGP requires no initiators, catalysts, or ligands, and polymer brushes can be realized in a one-step reaction at room temperature under UV irradiation. ${ }^{31}$

Received: December 4, 2019

Accepted: January 30, 2020

Published: January 30, 2020 
On the basis of the above facts, we now report a facile method for the functionalization of gCN by SIPGP using various vinyl monomers. As gCN bears amine groups, hydrogen atoms from these groups can be abstracted under UV irradiation. These radicals can then trigger surface-initiated radical polymerization of a variety of vinyl monomers such as methacrylates, acrylamides, and styrene. The gCN-polymer brush composites show good dispersibility in different solvents (water, ethanol, and tetrahydrofuran (THF)). Patterned polymer brushes on gCN can be realized by employing photomask and microcontact printing technology. Furthermore, polymer brushes with incorporated silver nanoparticles on $\mathrm{gCN}$ can potentially be applied as an active layer for surface-enhanced Raman spectroscopy (SERS). We demonstrated this application with repeatable usage at least five times and its outstanding photocatalytic degradation performance of rhodamine 6G (Rh6G) dyes under visible light conditions. This method provides a new way for the functionalization of $\mathrm{gCN}$ with tailorable properties, broadening its applications as a promising material for sensing and environmental remediation.

\section{EXPERIMENTAL SECTION}

2.1. Materials and Characterizations. Melamine, silver nitrate $\left(\mathrm{AgNO}_{3}, 99.99 \%\right)$, sodium borohydride $\left(\mathrm{NaBH}_{4}\right)$, Rh6G (99\%), styrene, 3-sulfopropyl methacrylate potassium salt (SPMA), N,Ndimethylaminoethyl methacrylate (DMAEMA), methylmethacrylate (MMA), 2-(methacryloyloxy)ethyl trimethylammonium chloride (METAC), and $\mathrm{N}$-isopropylacrylamide (NIPAm) were purchased from Sigma-Aldrich. Before polymerization, the inhibitors in the monomers were removed by passing the monomers through aluminum oxide (Sigma-Aldrich). Toluene, acetone, THF, ethanol, isopropanol (IPA), and methanol of analytical grade were obtained from Aldrich and used as received. Millipore water was used throughout the experiment. Si wafers with a $300 \mathrm{~nm}$ oxide layer were used for all depositions.

Thermogravimetric analysis (TGA) data were obtained using a thermal analyzer (TGA1 SF/1100 XP1U, METTLER TOLEDO, Switzerland) from room temperature to $1000{ }^{\circ} \mathrm{C}$ at a heating rate of 5 ${ }^{\circ} \mathrm{C} \min ^{-1}$ in a nitrogen gas atmosphere. X-ray photoelectron spectroscopy (XPS) measurements were performed using a Kratos Axis UltraDLD spectrometer (Kratos Analytical-A Shimadzu Group Company) with a monochromatic Al-K $\alpha$ source $(1486.6 \mathrm{eV})$. Fourier transform infrared spectroscopy (FTIR) spectrum was recorded using a Spectrum 100 (Perkin Elmer, Inc.) spectrometer, and a scan range of $4000-500 \mathrm{~cm}^{-1}$ was collected. X-ray diffraction (XRD) was conducted on a Bruker D8 Advance instrument in Bragg-Brentano mode with $\mathrm{Cu} \mathrm{K} \alpha$ radiation $(1.54 \AA, 40 \mathrm{kV}$ and $40 \mathrm{~mA}$ ). Atomic force microscopy (AFM, NT-MDT, Russia) was used to image the morphology and the surface roughness (root mean square (RMS)) of $\mathrm{gCN}$ and polymer brush-modified gCN. The size and morphology of the samples were determined with a scanning electron microscope (SEM, Zeiss Gemini 500, ZEISS company, Germany). Contact angles were measured using the Drop Shape Analysis System DSA10 from Krüss, and drop volumes were $1 \mu \mathrm{L}$. Ultraviolet-visible (UV-Vis) spectra were recorded on a Cary 4000 (Varian) photometer equipped with a Praying-MantisTM unit (Harrick), and a scan range of 190$800 \mathrm{~nm}$ was employed. The sample powders were pulverized with $\mathrm{BaSO}_{4}$ (white standard DIN 5033, Merck) and pressed into disks. Raman spectra were recorded using an NTEGRA Spectra system (laser: $532 \mathrm{~nm}, \mathrm{NT}-\mathrm{MDT}$, Moscow, Russia).

2.2. Methods. 2.2.1. Synthesis of $g C N$. Graphitic carbon nitride $(\mathrm{gCN})$ was prepared by thermal oxidation etching in air. ${ }^{32}$ Briefly, melamine was heated to $550{ }^{\circ} \mathrm{C}$ for $4 \mathrm{~h}$ in air at a heating rate of 2.3 ${ }^{\circ} \mathrm{C} \mathrm{min}^{-1}$. The obtained yellow agglomerates were milled into the powder with a mortar. Then, this powder was heated in an open ceramic container at a heating rate of $5{ }^{\circ} \mathrm{C} \mathrm{min}-1$ to $500{ }^{\circ} \mathrm{C}$ and kept at this temperature for $2 \mathrm{~h}$. Finally, the gCN was obtained (yield: $3 \%$ ) and characterized.

2.2.2. Preparation of Homogeneous Dispersion of $g C N$. The exfoliation of gCN powders was achieved by ultrasonication in IPA. The obtained light-yellow dispersion was centrifuged (SIGMA 3K30, $2000 \mathrm{rpm}, 10 \mathrm{~min}$ ) to remove bigger size particles of $\mathrm{gCN}$. After concentration and drying of the suspension, the obtained $\mathrm{gCN}$ powders were redispersed into anhydrous ethanol, followed by ultrasonication to obtain homogeneous suspensions.

2.2.3. Spin-Coating of $g C N$ Films. $\mathrm{Si} / \mathrm{SiO}_{2}$ wafers were first precleaned with a piranha solution $\left(\mathrm{H}_{2} \mathrm{SO}_{4}: \mathrm{H}_{2} \mathrm{O}_{2}=3: 1 \mathrm{vol} / \mathrm{vol}\right)$ at 90 ${ }^{\circ} \mathrm{C}$ for $45 \mathrm{~min}$. Then, the wafers were cleaned and rinsed by sequential ultrasonication in distilled water, acetone, and ethanol, successively. Subsequently, the wafers were dried under a flow of nitrogen. The gCN ethanol solution was drop-coated onto precleaned $\mathrm{Si} / \mathrm{SiO}_{2}$ wafer, and then the wafer was accelerated to a spin speed of 1500 $\mathrm{rpm}$ for a spinning time of $30 \mathrm{~s}$. The spin-coated films were dried in vacuum at $80{ }^{\circ} \mathrm{C}$ for $12 \mathrm{~h}$. The surface morphology of films was imaged using AFM.

2.2.4. Microcontact Printing gCN Patterns. A poly(dimethylsiloxane) (PDMS) stamp was inked by soaking it in an ethanol solution of $\mathrm{gCN}$ for $3 \mathrm{~min}$ and dried with nitrogen. Next, the stamp was brought in contact with the $\mathrm{Si} / \mathrm{SiO}_{2}$ substrates for 1 min to fabricate the patterned $\mathrm{gCN}$ on the $\mathrm{Si} / \mathrm{SiO}_{2}$ substrates.

2.2.5. Preparation of Polymer Brushes. gCN powders $(20 \mathrm{mg}), 1$ $\times 1 \mathrm{~cm}^{2}$ of spin-coated gCN films, and patterned gCN films were submerged in the degassed monomer (i.e., styrene, MMA, DMAEMA, NIPAm, SPMA, and METAC) in a glass tube, which was tightly sealed and subsequently irradiated with UV light $(\lambda=365 \mathrm{~nm})$ and a total power density of $8 \mathrm{~mW} \mathrm{~cm}{ }^{-2}$. After photopolymerization, the samples were rinsed with different good solvents for different polymer brushes (polystyrene (PS): toluene; poly(methylmethacrylate) (PMMA): acetone; poly $(N, N$-dimethylaminoethyl methacrylate) (PDMAEMA): ethanol and water; poly $(N$-isopropylacrylamide) (PNIPAm): water; poly(3-sulfopropyl methacrylate) (PSPMA): water; poly (2-(methacryloyloxy)ethyl trimethylammonium chloride) (PMETAC): water) to remove the physisorbed polymers so that only covalently grafted polymer brushes remain. The samples were then dried under nitrogen flow. The final products were called as polymergrafted gCN (polymer-g-gCN).

2.2.6. Patterning of Polymer Brushes. On the top of a spin-coated $\mathrm{gCN}$ film, a photomask was fixed, and then the film put into a glass tube with the degassed monomer solution. Subsequently, the glass tube was sealed and irradiated with UV light for $2 \mathrm{~h}$. The samples were washed with good solvents (PDMAEMA: water and ethanol) according to the monomers used. The patterned polymer brushmodified gCN was achieved.

2.2.7. Synthesis of Silver Nanoparticles (AgNPs) in PDMAEMA Brush-Modified $g C N$ (AgNPs/PDMAEMA-g-gCN). An aqueous $\mathrm{AgNO}_{3}$ solution $(0.01 \mathrm{M})$ was degassed for 15 min under argon flow; then PDMAEMA-modified gCN substrates were submerged into the $\mathrm{AgNO}_{3}$ solution and stirred for $1 \mathrm{~h}$ under argon flow. Subsequently, the samples were quickly dipped and stirred in an aqueous $\mathrm{NaBH}_{4}$ solution $(0.2 \mathrm{M})$ for $10 \mathrm{~min}$. Finally, the samples were washed several times with water and dried in argon atmosphere.

2.2.8. Characterization of SERS Samples. To test the feasibility of the active SERS substrate, Rh6G was used as the Raman model probe. A drop of Rh6G solution in ethanol $\left(1 \times 10^{-6} \mathrm{M}\right)$ was dropped onto unmodified gCN, PDMAEMA- $g$-gCN, and AgNPs/PDMAEMA- $g$ $\mathrm{gCN}$ substrates. After drying under ambient conditions, all substrates were washed with deionized water and absolute ethanol several times to remove the free Rh6G molecules. Raman spectra were recorded by using a custom-made confocal Raman spectrometer (Ntegra Aura/ Spectra) from NT-MDT. All samples were excited with laser light of $532 \mathrm{~nm}$ wavelength and $2 \mathrm{~mW}$ laser power.

2.2.9. Photocatalytic Degradation Performance. The photocatalytic activity was evaluated by the photocatalytic degradation of the dye Rh6G on AgNPs/PDMAEMA-g-gCN substrates. The Rh6G/ AgNPs/PDMAEMA-g-gCN substrates were irradiated by an RPR4190A lamp with an irradiation distance of $5 \mathrm{~cm}$. Upon irradiation, 


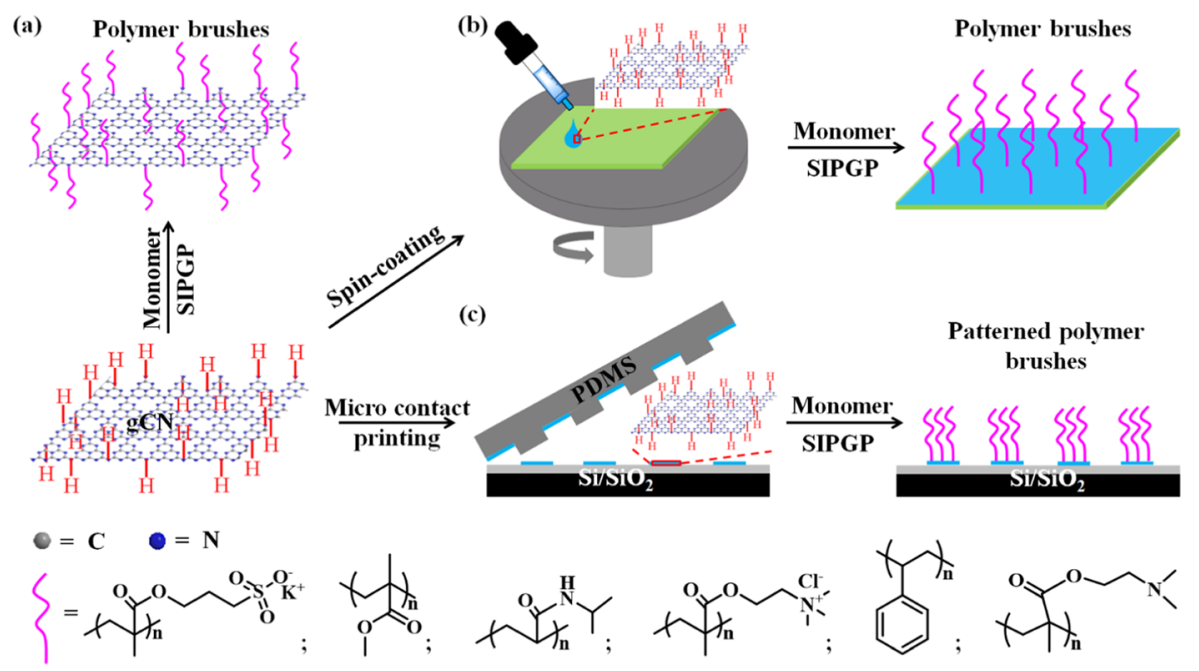

Figure 1. Schematic illustration of the preparation of polymer brushes on gCN particles (a), spin-coated gCN film (b), and microcontact printing gCN patterns (c).
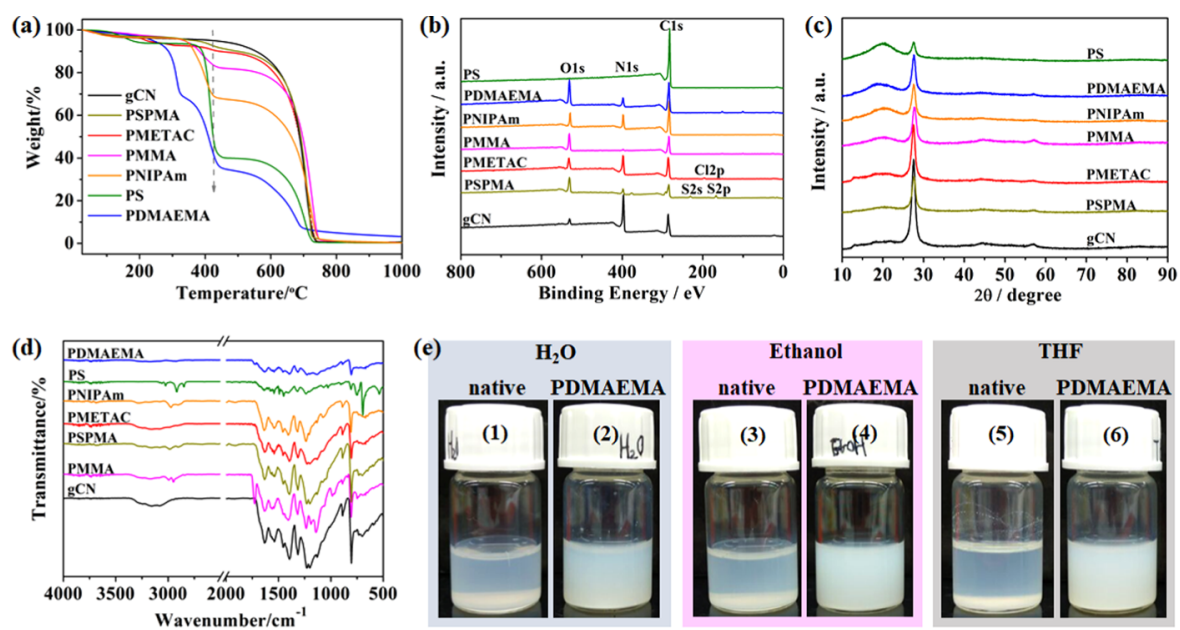

Figure 2. (a) TGA curves, (b) XPS spectra, (c) XRD patterns, and (d) FTIR spectra of native gCN and polymer brush-modified gCN. (e) The dispersibility of gCN $(1,3,5)$ and PDMAEMA-g-gCN $(2,4,6)$ in $\mathrm{H}_{2} \mathrm{O}$, ethanol, and THF after 3 months, respectively. The concentrations of gCN and PDMAEMA-g-gCN in solvent are both $3 \mathrm{mg} \mathrm{mL}$.

the substrates were moved and measured by Raman at certain time intervals up to $6 \mathrm{~h}$. Then the SERS spectra of Rh6G were recorded using a custom-made confocal Raman spectrometer (Ntegra Aura/ Spectra) from NT-MDT.

\section{RESULTS AND DISCUSSION}

3.1. Functionalization of gCN with Polymer Brushes. $\mathrm{gCN}$ was first obtained through the polycondensation of melamine, as reported previously. ${ }^{33} \mathrm{Next}$, gCN was immersed into a monomer solution. The surface-initiated polymerization of vinyl monomers by SIPGP was performed by the irradiation of $\mathrm{gCN}$ in a degassed bulk monomer with UV light $(W=\sim 8$ $\left.\mathrm{mW} \mathrm{cm} \mathrm{cm}^{-2}, \lambda_{\max }=365 \mathrm{~nm}\right)$. During the UV-induced photografting reaction process, an $\mathrm{H}$-terminated surface is required to facilitate a radical mechanism. ${ }^{34}$ Various researchers proved the success of grafting polymer brushes on H-terminated substrates. ${ }^{24,29,33}$ The terminal amino function is the most effective surface function for SIPGP because of the low $\mathrm{N}-\mathrm{H}$ bond dissociation energy. ${ }^{36,37} \mathrm{NH} /$ $\mathrm{NH}_{2}$ groups exist in $\mathrm{gCN}$, as shown by theoretical calculations and experimental observations. ${ }^{38}$ Hydrogen atoms from these groups can be abstracted to start a free radical surface-initiated polymerization, which leads to the formation of polymer brushes. ${ }^{23,34}$

Figure 1 shows a schematic illustration for the preparation of gCN-polymer composites. The feasibility and generality of our method are demonstrated here with the grafting and subsequent characterization of various polymer brushes on gCN, including PDMAEMA, PSPMA, PMETAC, PNIPAm, PMMA, and PS. Besides, polymer brushes on gCN particles (Figure 1a), spin-coated gCN films (Figure 1b), and microcontact printing $(\mu \mathrm{CP}) \mathrm{gCN}$ patterns (Figure 1c) were also prepared to give a robust demonstration of successful grafting.

3.2. Characterization of Polymer Brush-Modified $\mathrm{gCN}$. The pristine and polymer-modified $\mathrm{gCN}$ were characterized by TGA, XPS, XRD, and FTIR. Before all characterizations and performance tests, the samples were washed rigorously several times with good solvents for each polymer brush, to ensure the complete removal of ungrafted monomers and untethered polymers. TGA was first conducted to calculate the weight ratios of polymer brushes grafted on $\mathrm{gCN}$ (Figure 2a and Table S1). For pristine $\mathrm{gCN}$, 

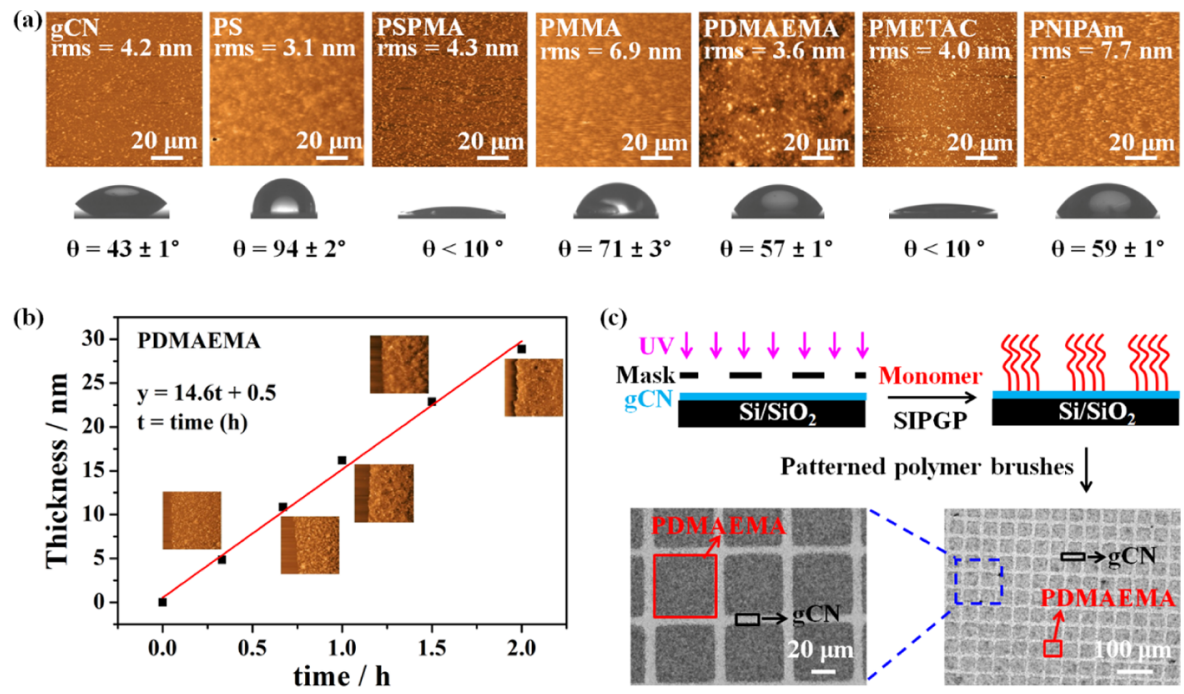

Figure 3. (a) AFM images and water contact angle of gCN and polymer-modified gCN. (b) Thickness evolution of polymer brushes grafted on $\mathrm{gCN}$ as a function of polymerization time for PDMAEMA brushes (PDMAEMA as a model system). (c) Schematic illustration and optical photographs of patterned PDMAEMA brushes on a spin-coated gCN film.

decomposition begins at $550{ }^{\circ} \mathrm{C}$ and almost finishes at around $750{ }^{\circ} \mathrm{C}$ whereas polymer brush-functionalized $\mathrm{gCN}$ shows some weight loss between 200 and $500{ }^{\circ} \mathrm{C}$ due to the decomposition of the grafted polymer brushes. For instance, PDMAEMA-grafted-gCN (PDMAEMA-g-gCN) clearly exhibits a two-stage weight loss (between 200 and $500{ }^{\circ} \mathrm{C}$ ). ${ }^{28}$ The TGA results confirm that the polymer induced by surface functionalization was covalently attached to the gCN rather than physically adsorbed. The amount of grafted polymer brushes on gCN depends on the type of monomer and polymerization time. XPS measurements were conducted to further confirm the success of polymer grafting on gCN. Figure $2 \mathrm{~b}$ shows a small but characteristic oxygen peak in the XPS survey spectrum of gCN (black curve), which indicates that the gCN absorbs a small amount of water from air. Compared to the pristine $\mathrm{gCN}$, the corresponding spectra of polymergrafted gCN display different elemental signals from each functional group in polymer brushes. For anionic PSPMA, the presence of the $S 2 \mathrm{p}$ peak at $168.1 \mathrm{eV}$ and the significantly larger $\mathrm{O} 1 \mathrm{~s}$ peak at $535.9 \mathrm{eV}$ demonstrate the successful polymerization of SPMA on gCN. For cationic PMETAC, the success of the polymerization is evidenced by the appearance of the $\mathrm{Cl} 2 \mathrm{p}$ peak at $196.5 \mathrm{eV}$, the weak $\mathrm{N} 1$ s peak at $402.1 \mathrm{eV}$, and the strong $\mathrm{O} 1 \mathrm{~s}$ peak at $532.7 \mathrm{eV}$ observed on the surface of PMETAC-g-gCN. For PS- $g$-gCN, the C 1 s peak at $285.2 \mathrm{eV}$ was observed, which could be due to the high weight ratio of PS in agreement with TGA results. XPS also confirmed the grafting of other polymers, such as PMMA, PNIPAm, and cationic PDMAEMA, on gCN by SIPGP. Figure $2 \mathrm{c}$ shows that $\mathrm{gCN}$ has the strongest $\mathrm{XRD}$ peak at $27.7^{\circ}$ due to the stacking reflection of the conjugated aromatic system, as in graphite materials. $^{[3 a]}$ Remarkably enough, after modification, the position of this (002) peak shows very slight shifts due to polymer embedding. This XRD observation, in addition to supporting our hypothesis, is consistent with SEM results. In FTIR spectra (Figure 2d), the chemical structures of gCN and polymer-modified gCN were further revealed. For example, in the spectrum of PMMA-g-gCN, the strong absorption peak at $1730 \mathrm{~cm}^{-1}$ can be attributed to absorption by carboxylic groups. The absorption peaks in the region $2800-3000 \mathrm{~cm}^{-1}$ correspond to the $-\mathrm{CH}_{2}$ and $-\mathrm{CH}_{3}$ groups. Combining the results from SEM and FTIR spectra (detailed discussion in Figures S1 and S2, in the Supporting Information) verifies that various types of polymers were successfully grafted onto $\mathrm{gCN}$. Moreover, the optical properties of $\mathrm{gCN}$ and polymer-modified gCN were investigated by UV-vis absorption spectroscopy (Figure S3). Compared with the absorption edge of pristine $\mathrm{gCN}$, the bandgap of polymer-modified gCN changes only slightly (Table S2), which confirms that the physical properties did not change by surface functionalization. This result encourages the application of functionalized $\mathrm{gCN}$ in chemical sensors and biosensors.

$\mathrm{Up}$ to now, the dispersion of $\mathrm{gCN}$ in any solvent remains one of the biggest challenges to its potential applications. Some reports showed that gCN could be dispersed in water or other organic solvents by extensive sonication, ${ }^{16,39}$ but this comes at the cost of long processing time and destruction of the sample's structure. In this work, the dispersibility of gCN was also investigated. Unmodified (native) gCN and PDMAEMA$g$-gCN were suspended in various solvents $\left(\mathrm{H}_{2} \mathrm{O}\right.$, ethanol, and THF) at the same concentration of $3 \mathrm{mg} \mathrm{mL}^{-1}$ at room temperature. As shown in Figure $2 \mathrm{e}$, the pristine $\mathrm{gCN}$ precipitates at the bottom of the vials, whereas PDMAEMA$g$-gCN shows good dispersion in various solvents even after 3 months of immersion. This demonstrates the effects of polymer modification on the gCN surface and the improved stability in suspension.

To further explore and demonstrate the surface properties of polymer-modified $\mathrm{gCN}$, a spin-coated $\mathrm{gCN}$ film was also used to graft polymer brushes. The surface morphology and thickness as well as the wettability of the pristine and polymer brushes modified gCN were characterized by AFM and water contact angle, respectively. Figure 3a shows the AFM images of pristine gCN and after SIPGP with different polymer brushes and their corresponding water contact angles. The spin-coated $\mathrm{gCN}$ film is inhomogeneous due to the nonuniform size of gCN nanoparticles. However, this inhomogeneity does not affect the grafting of polymer brushes on the gCN films. The surface roughness (RMS) of gCN is $4.2 \mathrm{~nm}$, whereas the RMS of PS, PSPMA, PMMA, PDMAEMA, PMETAC, and PNIPAm 


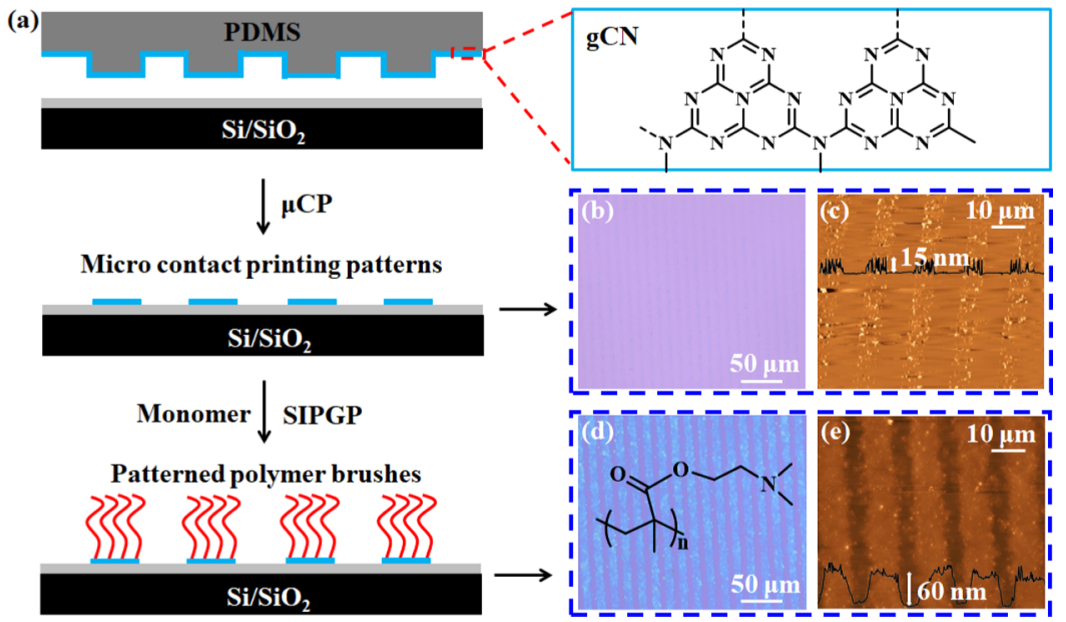

Figure 4. (a) Schematic illustration of preparing polymer brushes on gCN surfaces by microcontact printing. (b, c) Optical image and AFM image of the patterned gCN by microcontact printing, respectively. (d, e) Optical image and AFM image of polymer brush-modified patterned gCN, respectively.

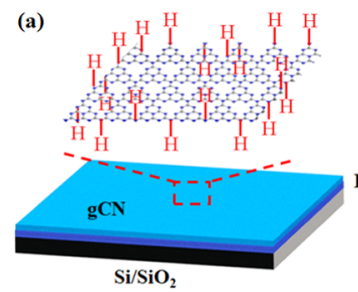

(b)

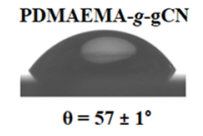

AgNPs/PDMAEMA-g-gCN
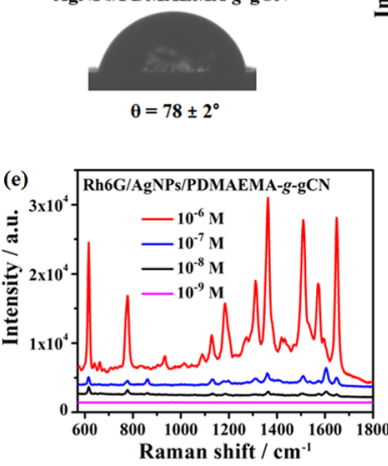
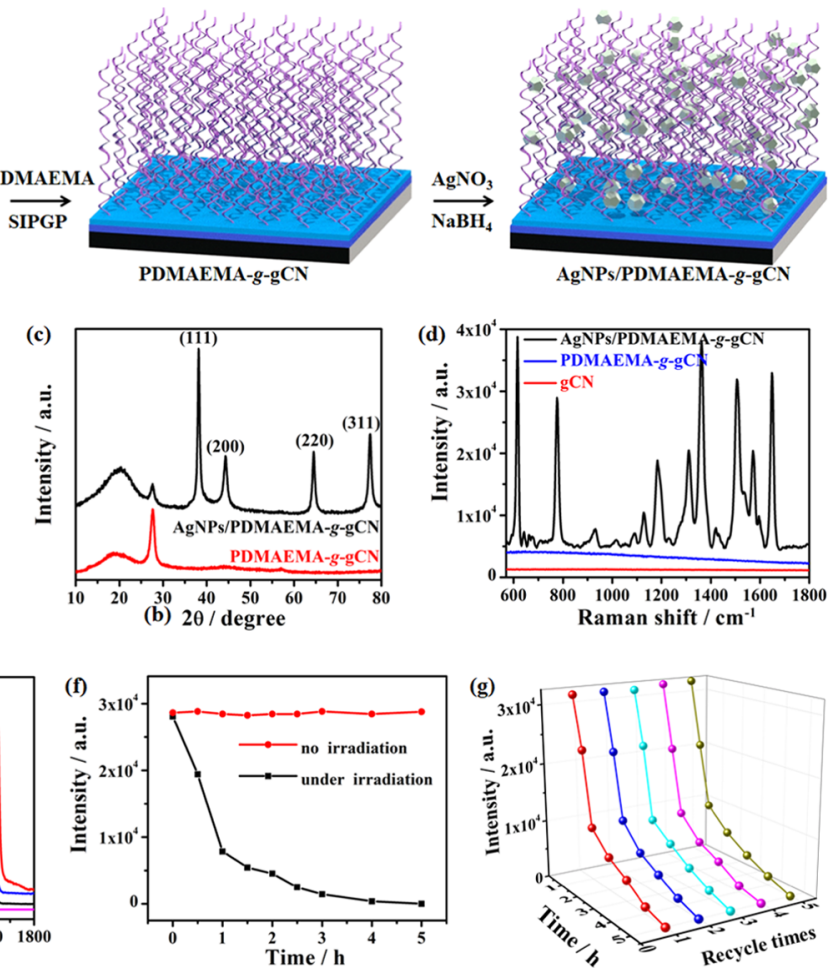

Figure 5. (a) Schematic illustration of the fabrication of PDMAEMA-g-gCN by SIPGP and subsequent immobilization of AgNPs on PDMAEMAg-gCN. (b) Contact angles and (c) XRD spectra of PDMAEMA-g-gCN and AgNPs/PDMAEMA-g-gCN. (d) SERS spectra of $10^{-6} \mathrm{M}$ Rh6G on native, PDMAEMA-g-gCN, and AgNPs/PDMAEMA-g-gCN. (e) SERS spectra for AgNPs/PDMAEMA-g-gCN probed with different concentrations of Rh6G from $10^{-6}$ to $10^{-9} \mathrm{M}$ in ethanol. (f) SERS intensity plot for the band at $1649 \mathrm{~cm}^{-1}$ vs time without and with visible light irradiation to completely remove the Rh6G from the AgNPs/PDMAEMA- $g$-gCN layer. (g) The SERS intensities at $1649 \mathrm{~cm}^{-1}$ for visible light-assisted degradation of $10^{-6} \mathrm{M} \mathrm{Rh6G}$ on AgNPs/PDMAEMA-g-gCN for repeatable usage up to five times.

are $3.1,4.3,6.9,3.6,4.0$, and $7.7 \mathrm{~nm}$, respectively, indicating the change in surface morphology after polymerization with the respective monomer. By utilizing this grafting method, the wettability of gCN can be easily tuned employing the abovementioned polymer brushes with different wetting behaviors. Contact angle (CA) measurements were performed to characterize the wettability of gCN before and after polymer brush modification (Figure 3a, lower images). The CA of gCN was $43 \pm 1^{\circ}$ and decreased to $<10^{\circ}$ after the grafting of METAC and SPMA, ${ }^{29}$ indicating that the surface became more hydrophilic. However, after the grafting of DMAEMA and NIPAm, CA values increased to $57 \pm 1$ and $59 \pm 1^{\circ}$, respectively, showing that the surfaces became less hydrophilic. After the grafting of PMMA and PS, the surfaces became more hydrophobic with CA values of $71 \pm 3$ and $94 \pm 2^{\circ}$, respectively. ${ }^{40}$ This wettability analysis shows that SIPGP can endow hydrophobicity/hydrophilicity to gCN and that the surface properties can be tuned by polymer brushes expanding the possibilities for $\mathrm{gCN}$ applications. Moreover, the height dependency on polymerization time for PDMAEMA was also 
studied. Figure $3 \mathrm{~b}$ shows a linear relationship between the thickness of polymer brushes and polymerization time. PDMAEMA has a high growth rate $\left(14.6 \mathrm{~nm} \mathrm{~h}^{-1}\right)$ on the gCN film due to similar wettability of gCN and DMAEMA. ${ }^{41}$ Furthermore, patterned polymer brushes on the gCN film can be realized by direct photopatterning using a mask on the top of the gCN film (Figure 3c). The optical microscopy images show that polymer brushes are only grafted on the areas where gCN was exposed to UV light. The well-defined microstructures confirm the simplicity of the direct patterning and selectivity of photopolymerization.

3.3. Microcontact Printing Patterns. Microcontact printing $(\mu \mathrm{CP})$ is one of the most attractive lithographic tools to get predefined patterns on substrates. ${ }^{42}$ Recently, Chen and co-workers used the $\mu \mathrm{CP}$ technology to obtain micropatterned graphene oxide films. ${ }^{43}$ Similarly, patterned gCN could also be prepared by $\mu \mathrm{CP}$, where PDMS and gCN are used as $\mu \mathrm{CP}$ stamps and inks, respectively, and with further pattern amplification by SIPGP, as shown schematically in Figure 4a. Herein, patterned gCN nanosheets were first stamped on the $\mathrm{Si} / \mathrm{SiO}_{2}$ substrate by $\mu \mathrm{CP}$. Subsequently, PDMAEMA brushes grown on the patterned gCN by SIPGP resulted in micropatterned polymer brushes. $\mu \mathrm{CP}$ is unique for providing well-defined microarchitectures with high stability even after grafting because gCN micropatterns with high stability can be generated on a $\mathrm{SiO}_{2}$ surface via hydrogen bonding interactions. ${ }^{44}$ Optical and AFM images were employed to compare patterned $\mathrm{gCN}$ and patterned polymer-modified gCN. Figure $4 \mathrm{a}$ shows the schematic illustration of making polymer brushes on patterned gCN by $\mu \mathrm{CP}$. PDMS and gCN are used as $\mu \mathrm{CP}$ stamps and inks, respectively. Figure $4 \mathrm{~b}$ shows high-quality line-shaped gCN arrays $10 \mu \mathrm{m}$ wide and $15 \mathrm{~nm}$ thicknesses, as obtained with an optical microscope. Figure $4 c$ shows the zoomed-in AFM height image of areas in Figure $4 \mathrm{~b}$ and reveals the same patterns as the optical image in Figure $4 \mathrm{~b}$. Subsequently, PDMAEMA brushes grown on the patterned gCN by SIPGP result in micropatterned polymer brushes with the thickness of about $60 \mathrm{~nm}$. Figure $4 \mathrm{~d}$ shows the optical image of patterned PDMAEMA brushes, and Figure 4e shows the AFM height which reveals the same pattern as observed in Figure $4 \mathrm{~d}$. The $\mu \mathrm{CP}$ can extend the potential applications of gCN such as studies of protein adsorption and cell attachment. ${ }^{45}$

3.4. Surface-Enhanced Raman Spectroscopy and Photocatalytic Degradation of Polymer Brush-Grafted gCN. Surface-enhanced Raman spectroscopy is a highly sensitive analytical tool to detect organic molecules with an extraordinary sensitivity down to single-molecule detection. ${ }^{46-48}$ To further demonstrate the potential application of polymer-functionalized gCN, SERS-active sensing substrates were fabricated by immobilizing AgNPs on PDMAEMA-g$\mathrm{gCN}$. Figure 5a shows the schematic illustration of the AgNPs/ PDMAEMA-g-gCN fabrication. PDMAEMA brushes were first grafted on the gCN layer through SIPGP. Subsequently, AgNPs were immobilized within the PDMAEMA brushes by the reduction of coordinated $\mathrm{Ag}^{+}$in an aqueous $\mathrm{NaBH}_{4}$ solution. Contact angle measurement, XRD analysis, and SEM confirmed the presence of AgNPs within the PDMAEMA brushes (Figures $5 b, c$ and S4). Figure $5 b$ shows that the $C A$ of PDMAEMA- $g$-gCN brushes and AgNPs/PDMAEMA- $g$-gCN is $57 \pm 1$ and $78 \pm 2^{\circ}$, respectively. The rise in CA can be related to the increase in brush surface roughness due to the presence of AgNPs. ${ }^{31,49}$ XRD patterns in Figure $5 \mathrm{c}$ confirm the crystalline structure of $\mathrm{AgNPs},{ }^{50}$ as a consequence of the successful AgNP synthesis with an average AgNP size of about $10 \mathrm{~nm}$ (Figure S4b). Figure S4c shows the homogeneous distribution of $\mathrm{Ag}$ confirming the uniform immobilization of AgNPs on PDMAEMA brushes.

The SERS performance of AgNPs/PDMAEMA- $g$-gCN was evaluated by measuring the Raman spectra of Rh6G (Figure $5 \mathrm{~d}$ ). Rh6G is commonly used as a Raman probe in SERS because this dye has a large Raman cross-section that generates a strong SERS signal. To evaluate the SERS enhancement of samples, native gCN, PDMAEMA-g-gCN, and AgNPs/ PDMAEMA-g-gCN layer substrates were first immersed in a $1 \times 10^{-6} \mathrm{M}$ Rh6G ethanol solution for $2 \mathrm{~h}$, subsequently rinsed with ethanol, and dried under nitrogen flow. The AgNPs/ PDMAEMA-g-gCN exhibits a remarkably high SERS signal, whereas no distinct Raman signal whatsoever is detected for native $\mathrm{gCN}$ or PDMAEMA-g-gCN.

The SERS spectra of Rh6G with different concentrations from $10^{-6}$ to $10^{-9} \mathrm{M}$ are shown in Figure 5e. The bands at around $616(\mathrm{C}-\mathrm{C}-\mathrm{C}$ in-plane bending vibration), $778(\mathrm{C}-\mathrm{H}$ out-of-plane bending vibration), 1184 (aromatic $\mathrm{C}-\mathrm{H}$ bend mode), and $1312 \mathrm{~cm}^{-1}$ (C-O-C stretching), and the $\mathrm{C}-\mathrm{C}$ stretching modes of Rh6G at 1364, 1511, 1574, and $1649 \mathrm{~cm}^{-1}$ are clearly observed. ${ }^{51,52}$ As the concentration of Rh6G decreases, consequently, the SERS intensity of Rh6G at 1649 $\mathrm{cm}^{-1}$ also decreases. Despite this decrease, even when the concentration of Rh6G reaches $10^{-8} \mathrm{M}$, the Raman signal of the dye molecule is still clearly detectable. This decrease in the SERS signal is due to the reduction in the Rh6G concentration, resulting in fewer target molecules adsorbed on the AgNPs/ PDMAEMA-g-gCN. The enhancement factor (EF) was calculated according to the following equation ${ }^{53}$

$$
\mathrm{EF}=\frac{I_{\mathrm{SERS}} C_{0}}{I_{0} C_{\mathrm{SERS}}}
$$

where $I_{\text {SERS }}$ and $C_{\text {SERS }}$ are the intensity of the Raman band at $1649 \mathrm{~cm}^{-1}$ and the concentration of Rh6G under SERS, respectively, $C_{0}$ is the concentration Rh6G, and $I_{0}$ is the intensity of the Raman band at $1649 \mathrm{~cm}^{-1}$ under normal Raman conditions (Figure S5). According to eq 1, the EF was calculated to be $2.4 \times 10^{7}$, indicating that the AgNPs/ PDMAEMA-g-gCN hybrid materials can work effectively as an SERS active layer for sensing applications.

Photocatalytic properties play a critical role in the clean energy sources, ${ }^{54}$ to further demonstrate the applicability of our hybrid AgNPs/PDMAEMA-g-gCN, and motivated by the widespread investigation of gCN as a visible-light-driven photocatalyst, ${ }^{5,55-68}$ we performed a typical photocatalytic degradation experiment under visible light irradiation. For this purpose, the photocatalytic degradation performance of Rh6G from Rh6G/AgNPs/PDMAEMA-g-gCN substrates under irradiation of visible light (RPR-4190A lamp) was investigated. The intensity of Rh6G decreased sharply after $1 \mathrm{~h}$ and vanished after $5 \mathrm{~h}$ of irradiation (Figure S6), indicating the complete decomposition of Rh6G in the AgNPs/PDMAEMA$g$-gCN substrates while preserving its photocatalytic functionality. To further elucidate the photodegradation performance, the Rh6G SERS peak intensity at $1649 \mathrm{~cm}^{-1}$ was plotted against irradiation time (Figure $5 \mathrm{f}$ ). The resulted plots show that the Rh6G was degraded with increasing irradiation time. This inspired us to study the reusability and stability of AgNPs/PDMAEMA-g-gCN structures. Therefore, sequential 
SERS and photodegradation experiments were conducted. As shown in Figure $5 \mathrm{~g}$, the AgNPs/PDMAEMA-g-gCN sample shows almost the same SERS intensity and relatively high photodegradation activity for five recycle times. The XRD patterns of the AgNPs/PDMAEMA-g-gCN samples before and after five reuse times show no structural changes (Figure S7), which demonstrates the excellent stability and the recyclability of AgNPs/PDMAEMA-g-gCN. AgNPs/PDMAEMA-g-gCN can be exploited as an SERS active layer to in situ monitor the degradation of pollutants or pharmaceutical waste.

\section{CONCLUSIONS}

In summary, we have presented an easy way to direct covalently bind various polymer brushes on gCN via SIPGP. The results indicate that SIPGP does not damage or alter the original structure of gCN. The polymer brush-grafted gCN exhibits a well-improved dispersibility in various solvents. Patterned polymer brushes on gCN can also be easily realized by employing $\mu \mathrm{CP}$. The AgNPs/PDMAEMA-g-gCN structure shows strong SERS enhancement with a surface enhancement factor of $2.4 \times 10^{7}$ for the detection of the Rh6G dye. Moreover, the complete removal of Rh6G via the photocatalytic degradation process on AgNPs/PDMAEMA-g-gCN under the irradiation of visible light exhibits the stability and good photocatalytic activity of the polymer brush-grafted $\mathrm{gCN}$. These results demonstrate that the polymer brushes with incorporated AgNPs on gCN can act as a recyclable active sensing layer for consecutive cycles of SERS and photocatalytic degradation processes. This multifunctionality has implications for applications in in situ monitoring of pollutants, such as dyes or pharmaceutical waste, with high chemical sensitivity as well as wastewater remediation. Therefore, SIPGP provides a powerful new set of tools for the covalent functionalization of gCN and may enable new applications in the field of catalysis, biosensors, and, most interestingly, environmental remediation, making an advantageous multifunctional platform.

\section{ASSOCIATED CONTENT}

\section{s) Supporting Information}

The Supporting Information is available free of charge at https://pubs.acs.org/doi/10.1021/acsami.9b21984.

Additional analytical data and information (weight ratio of polymer brushes); SEM images, FTIR spectra, UVvis absorption spectrum, and bandgap energy of gCN before and after polymerization with various polymer brushes; Raman spectrum of Rh6G in water; Raman spectra of Rh6G on AgNPs/PDMAEMA-g-gCN under visible light irradiation; XRD patterns of AgNPs/ PDMAEMA- $g$-gCN before and after five reuse times under visible light irradiation (PDF)

\section{AUTHOR INFORMATION}

\section{Corresponding Authors}

Raul D. Rodriguez - Research School of Chemistry and Applied Biomedical Sciences, Tomsk Polytechnic University, 634050 Tomsk, Russia; Email: raul@tpu.ru

Rainer Jordan - Chair of Macromolecular Chemistry, Faculty of Chemistry and Food Chemistry, School of Science, Technische Universität Dresden, 01069 Dresden, Germany; 이이.org/ 0000-0002-9414-1597; Email: rainer.jordan@tu-dresden.de
Ihsan Amin - Chair of Macromolecular Chemistry, Faculty of Chemistry and Food Chemistry, School of Science, Technische Universität Dresden, 01069 Dresden, Germany; Van't Hoff Institute of Molecular Science, University of Amsterdam, 1098 XH Amsterdam, The Netherlands; O orcid.org/0000-00027354-4717; Email: i.amin@uva.nl

\section{Authors}

Wenbo Sheng - Chair of Macromolecular Chemistry, Faculty of Chemistry and Food Chemistry, School of Science, Technische Universität Dresden, 01069 Dresden, Germany; Leibniz Institute of Polymer Research Dresden e.V., 01069 Dresden, Germany

Wei Li - Chair of Macromolecular Chemistry, Faculty of Chemistry and Food Chemistry, School of Science, Technische Universität Dresden, 01069 Dresden, Germany

Deming Tan - Department of Inorganic Chemistry, Technische Universität Dresden, 01069 Dresden, Germany

Panpan Zhang - Chair of Molecular Functional Materials, Faculty of Chemistry and Food Chemistry, School of Science, Technische Universität Dresden, 01069 Dresden, Germany

En Zhang - Department of Inorganic Chemistry, Technische Universität Dresden, 01069 Dresden, Germany

Evgeniya Sheremet - Research School of Physics, Tomsk Polytechnic University, 634050 Tomsk, Russia

Bernhard V.K.J. Schmidt - School of Chemistry, University of Glasgow, Glasgow G12 8QQ, U.K.

Xinliang Feng - Chair of Molecular Functional Materials, Faculty of Chemistry and Food Chemistry, School of Science, Technische Universität Dresden, 01069 Dresden, Germany; () orcid.org/0000-0003-3885-2703

Complete contact information is available at: https://pubs.acs.org/10.1021/acsami.9b21984

\section{Author Contributions}

The manuscript was written through contributions of all authors. All authors have given approval to the final version of the manuscript.

\section{Notes}

The authors declare no competing financial interest.

\section{ACKNOWLEDGMENTS}

W.S. and R.J. acknowledge the financial support from the Initiative and Networking Fund of the Helmholtz Association of German Research Centers through the International Helmholtz Research School for Nanoelectronic Networks, IHRS NANONET (VH-KO-606). W.S., P.Z., X.F., R.J., and I.A. thank to Center for Advancing Electronics Dresden (CfAED) for financial support. E.S. and R.D.R. acknowledge the support from Russian Science Foundation grant (Project Number 19-75-10046).

\section{REFERENCES}

(1) Chen, X.; Zhang, J.; Fu, X.; Antonietti, M.; Wang, X. Fe-g- $\mathrm{C}_{3} \mathrm{~N}_{4^{-}}$ Catalyzed Oxidation of Benzene to Phenol Using Hydrogen Peroxide and Visible Light. J. Am. Chem. Soc. 2009, 131, 11658-11659.

(2) Su, F.; Mathew, S. C.; Lipner, G.; Fu, X.; Antonietti, M.; Blechert, S.; Wang, X. mpg-C $\mathrm{C}_{3} \mathrm{~N}_{4}$-Catalyzed Selective Oxidation of Alcohols Using $\mathrm{O}_{2}$ and Visible Light. J. Am. Chem. Soc. 2010, 132, 16299-16301.

(3) Li, X. H.; Chen, J. S.; Wang, X.; Sun, J.; Antonietti, M. MetalFree Activation of Dioxygen by Graphene/g- $\mathrm{C}_{3} \mathrm{~N}_{4}$ Nanocomposites: 
Functional Dyads for Selective Oxidation of Saturated Hydrocarbons. J. Am. Chem. Soc. 2011, 133, 8074-8077.

(4) Su, F.; Mathew, S. C.; Mohlmann, L.; Antonietti, M.; Wang, X.; Blechert, S. Aerobic Oxidative Coupling of Amines by Carbon Nitride Photocatalysis with Visible Light. Angew. Chem., Int. Ed. 2011, 50, 657-660.

(5) Sun, J.; Schmidt, B. V. K. J.; Wang, X.; Shalom, M. Self-Standing Carbon Nitride-Based Hydrogels with High Photocatalytic Activity. ACS Appl. Mater. Interfaces 2017, 9, 2029-2034.

(6) Cheng, N.; Tian, J.; Liu, Q.; Ge, C.; Qusti, A. H.; Asiri, A. M.; Al-Youbi, A. O.; Sun, X. Au-Nanoparticle-Loaded Graphitic Carbon Nitride Nanosheets: Green Photocatalytic Synthesis and Application toward the Degradation of Organic Pollutants. ACS Appl. Mater. Interfaces 2013, 5, 6815-6819.

(7) Wang, X.; Maeda, K.; Thomas, A.; Takanabe, K.; Xin, G.; Carlsson, J. M.; Domen, K.; Antonietti, M. A Metal-Free Polymeric Photocatalyst for Hydrogen Production from Water under Visible Light. Nat. Mater. 2009, 8, 76-80.

(8) Liu, J.; Liu, Y.; Liu, N.; Han, Y.; Zhang, X.; Huang, H.; Lifshitz, Y.; Lee, S.-T.; Zhong, J.; Kang, Z. Metal-Free Efficient Photocatalyst for Stable Visible Water Splitting via a Two-Electron Pathway. Science 2015, 347, 970-974.

(9) Chen, X.; Randall, D. P.; Perruchot, C.; Watts, J. F.; Patten, T. E.; von Werne, T.; Armes, S. P. Synthesis and Aqueous Solution Properties of Polyelectrolyte-Grafted Silica Particles Prepared by Surface-Initiated Atom Transfer Radical Polymerization. J. Colloid Interface Sci. 2003, 257, 56-64.

(10) Yan, S. C.; Li, Z. S.; Zou, Z. G. Photodegradation Performance of g- $\mathrm{C}_{3} \mathrm{~N}_{4}$ Fabricated by Directly Heating Melamine. Langmuir 2009, 25, 10397-10401.

(11) Cui, Y.; Ding, Z.; Liu, P.; Antonietti, M.; Fu, X.; Wang, X. Metal-Free Activation of $\mathrm{H}_{2} \mathrm{O}_{2}$ by $\mathrm{g}^{-} \mathrm{C}_{3} \mathrm{~N}_{4}$ under Visible Light Irradiation for the Degradation of Organic Pollutants. Phys. Chem. Chem. Phys. 2012, 14, 1455-1462.

(12) Ong, W. J.; Tan, L. L.; Ng, Y. H.; Yong, S. T.; Chai, S. P. Graphitic Carbon Nitride $\left(\mathrm{g}-\mathrm{C}_{3} \mathrm{~N}_{4}\right)$-Based Photocatalysts for Artificial Photosynthesis and Environmental Remediation: Are We a Step Closer To Achieving Sustainability? Chem. Rev. 2016, 116, 71597329.

(13) Arazoe, H.; Miyajima, D.; Akaike, K.; Araoka, F.; Sato, E.; Hikima, T.; Kawamoto, M.; Aida, T. An Autonomous Actuator Driven by Fluctuations in Ambient Humidity. Nat. Mater. 2016, 15, 1084-1089.

(14) Tian, J.; Liu, Q.; Asiri, A. M.; Al-Youbi, A. O.; Sun, X. Ultrathin Graphitic Carbon Nitride Nanosheet: A Highly Efficient Fluorosensor for Rapid, Ultrasensitive Detection of $\mathrm{Cu}^{2+}$. Anal. Chem. 2013, 85, 5595-5599.

(15) Zhang, X.; Xie, X.; Wang, H.; Zhang, J.; Pan, B.; Xie, Y. Enhanced Photoresponsive Ultrathin Graphitic-Phase $\mathrm{C}_{3} \mathrm{~N}_{4}$ Nanosheets for Bioimaging. J. Am. Chem. Soc. 2013, 135, 18-21.

(16) Yang, S.; Gong, Y.; Zhang, J.; Zhan, L.; Ma, L.; Fang, Z.; Vajtai, R.; Wang, X.; Ajayan, P. M. Exfoliated Graphitic Carbon Nitride Nanosheets as Efficient Catalysts for Hydrogen Evolution under Visible Light. Adv. Mater. 2013, 25, 2452-2456.

(17) Kumru, B.; Antonietti, M.; Schmidt, B. V. K. J. Enhanced Dispersibility of Graphitic Carbon Nitride Particles in Aqueous and Organic Media via a One-Pot Grafting Approach. Langmuir 2017, 33, 9897-9906.

(18) Sun, J.; Phatake, R.; Azoulay, A.; Peng, G.; Han, C.; Barrio, J.; $\mathrm{Xu}$, J.; Wang, X.; Shalom, M. Covalent Functionalization of Carbon Nitride Frameworks through Cross-Coupling Reactions. Chem. - Eur. J. 2018, 24, 14921-14927.

(19) Cao, Q.; Kumru, B.; Antonietti, M.; Schmidt, B. V. K. J. Grafting Polymers onto Carbon Nitride via Visible-Light-Induced Photofunctionalization. Macromolecules 2019, 52, 4989-4996.

(20) Mitzi, D. B.; Yuan, M.; Liu, W.; Kellock, A. J.; Chey, S. J.; Deline, V.; Schrott, A. G. A High-Efficiency Solution-Deposited ThinFilm Photovoltaic Device. Adv. Mater. 2008, 20, 3657-3662.
(21) Xu, J.; Antonietti, M. The Performance of Nanoparticulate Graphitic Carbon Nitride as an Amphiphile. J. Am. Chem. Soc. 2017, 139, 6026-6029.

(22) Sheng, W.; Amin, I.; Neumann, C.; Dong, R.; Zhang, T.; Wegener, E.; Chen, W. L.; Forster, P.; Tran, H. Q.; Loffler, M.; Winter, A.; Rodriguez, R. D.; Zschech, E.; Ober, C. K.; Feng, X.; Turchanin, A.; Jordan, R. Polymer Brushes on Hexagonal Boron Nitride. Small 2019, 15, No. 1805228.

(23) Steenackers, M.; Lud, S. Q.; Niedermeier, M.; Bruno, P.; Gruen, D. M.; Feulner, P.; Stutzmann, M.; Garrido, J. A.; Jordan, R. Structured Polymer Grafts on Diamond. J. Am. Chem. Soc. 2007, 129, 15655-15661.

(24) Steenackers, M.; Gigler, A. M.; Zhang, N.; Deubel, F.; Seifert, M.; Hess, L. H.; Lim, C. H.; Loh, K. P.; Garrido, J. A.; Jordan, R.; Stutzmann, M.; Sharp, I. D. Polymer Brushes on Graphene. J. Am. Chem. Soc. 2011, 133, 10490-10498.

(25) Amin, I.; Steenackers, M.; Zhang, N.; Beyer, A.; Zhang, X.; Pirzer, T.; Hugel, T.; Jordan, R.; Gölzhäuser, A. Polymer Carpets. Small 2010, 6, 1623-1630.

(26) Amin, I.; Steenackers, M.; Zhang, N.; Schubel, R.; Beyer, A.; Gölzhäuser, A.; Jordan, R. Patterned Polymer Carpets. Small 2011, 7, 683-687.

(27) Gu, J.; Xiao, P.; Chen, J.; Liu, F.; Huang, Y.; Li, G.; Zhang, J.; Chen, T. Robust Preparation of Superhydrophobic Polymer/Carbon Nanotube Hybrid Membranes for Highly Effective Removal of Oils and Separation of Water-in-Oil Emulsions. J. Mater. Chem. A 2014, 2, $15268-15272$.

(28) Hwang, W. S.; Remskar, M.; Yan, R.; Protasenko, V.; Tahy, K.; Chae, S. D.; Zhao, P.; Konar, A.; Xing, H.; Seabaugh, A.; Jena, D. Transistors with Chemically Synthesized Layered Semiconductor WS Exhibiting $10^{5}$ Room Temperature Modulation and Ambipolar Behavior. Appl. Phys. Lett. 2012, 101, No. 013107.

(29) Sheng, W.; Li, B.; Wang, X.; Dai, B.; Yu, B.; Jia, X.; Zhou, F. Brushing up from "Anywhere" under Sunlight: a Universal SurfaceInitiated Polymerization from Polydopamine-Coated Surfaces. Chem. Sci. 2015, 6, 2068-2073.

(30) Sheng, W.; Li, W.; Yu, B.; Li, B.; Jordan, R.; Jia, X.; Zhou, F. Mussel-Inspired Two-Dimensional Freestanding Alkyl-Polydopamine Janus Nanosheets. Angew. Chem., Int. Ed. 2019, 58, 12018-12022.

(31) Gupta, S.; Agrawal, M.; Conrad, M.; Hutter, N. A.; Olk, P.; Simon, F.; Eng, L. M.; Stamm, M.; Jordan, R. Poly(2(dimethylamino)ethyl methacrylate) Brushes with Incorporated Nanoparticles as a SERS Active Sensing Layer. Adv. Funct. Mater. 2010, 20, 1756-1761.

(32) Li, W.; Zhang, G.; Sheng, W.; Dong, C.; Dai, Y.; Li, C.; Wang, R.; Shi, Y.; Guo, X.; Jia, X. Engineering Plasmonic Ag/AgClPolydopamine-Carbon Nitride Composites for Enhanced Photocatalytic Activity based on Mussel Chemistry. RSC Adv. 2016, 6, 106697-106704.

(33) Pakdel, A.; Zhi, C.; Bando, Y.; Golberg, D. Low-Dimensional Boron Nitride Nanomaterials. Mater. Today 2012, 15, 256-265.

(34) Zhang, N.; Steenackers, M.; Luxenhofer, R.; Jordan, R. BottleBrush Brushes: Cylindrical Molecular Brushes of Poly(2-oxazoline) on Glassy Carbon. Macromolecules 2009, 42, 5345-5351.

(35) Steenackers, M.; Sharp, I. D.; Larsson, K.; Hutter, N. A.; Stutzmann, M.; Jordan, R. Structured Polymer Brushes on Silicon Carbide. Chem. Mater. 2010, 22, 272-278.

(36) Zhang, N.; Pompe, T.; Amin, I.; Luxenhofer, R.; Werner, C.; Jordan, R. Tailored Poly(2-Oxazoline) Polymer Brushes to Control Protein Adsorption and Cell Adhesion. Macromol. Biosci. 2012, 12, 926-936.

(37) Hou, L.; Wang, L.; Zhang, N.; Xie, Z.; Dong, D. Polymer Brushes on Metal-Organic Frameworks by UV-Induced Photopolymerization. Polym. Chem. 2016, 7, 5828-5834.

(38) Zhang, J.; Zhang, M.; Lin, L.; Wang, X. Sol Processing of Conjugated Carbon Nitride Powders for Thin-Film Fabrication. Angew. Chem. 2015, 127, 6395-6399. 
(39) Xu, J.; Zhang, L.; Shi, R.; Zhu, Y. Chemical Exfoliation of Graphitic Carbon Nitride for Efficient Heterogeneous Photocatalysis. J. Mater. Chem. A 2013, 1, 14766-14772.

(40) Ma, S.; Zhang, X.; Yu, B.; Zhou, F. Brushing up Functional Materials. NPG Asia Mater. 2019, 11, No. 24.

(41) Hafner, D.; Ziegler, L.; Ichwan, M.; Zhang, T.; Schneider, M.; Schiffmann, M.; Thomas, C.; Hinrichs, K.; Jordan, R.; Amin, I. Mussel-Inspired Polymer Carpets: Direct Photografting of Polymer Brushes on Polydopamine Nanosheets for Controlled Cell Adhesion. Adv. Mater. 2016, 28, 1489-1494.

(42) Chen, T.; Amin, I.; Jordan, R. Patterned Polymer Brushes. Chem. Soc. Rev. 2012, 41, 3280-3296.

(43) Xiao, P.; Qiu, N.; Gu, J.; Wang, S.; He, J.; Huang, C. F.; Zhang, J.; Huang, Y.; Chen, T. Direct Supramolecular Interacted Graphene Oxide Assembly on Graphene as an Active and Defect-Free Functional Platform. Chem. Commun. 2017, 53, 1949-1952.

(44) Hou, L.; Zhou, M.; Dong, X.; Wang, L.; Xie, Z.; Dong, D.; Zhang, N. Controlled Growth of Metal-Organic Frameworks on Polymer Brushes. Chem. - Eur. J. 2017, 23, 13337-13341.

(45) Kosobrodova, E.; Gan, W. J.; Kondyurin, A.; Thorn, P.; Bilek, M. M. M. Improved Multiprotein Microcontact Printing on Plasma Immersion Ion Implanted Polystyrene. ACS Appl. Mater. Interfaces 2018, 10, 227-237.

(46) Kneipp, K.; Wang, Y.; Kneipp, H.; Perelman, L. T.; Itzkan, I.; Dasari, R. R.; Feld, M. S. Single Molecule Detection Using SurfaceEnhanced Raman Scattering (SERS). Phys. Rev. Lett. 1997, 78, No. 1667.

(47) Wang, L.; Li, H.; Tian, J.; Sun, X. Monodisperse, MicrometerScale, Highly Crystalline, Nanotextured Ag Dendrites: Rapid, LargeScale, Wet-Chemical Synthesis and Their Application as SERS Substrates. ACS Appl. Mater. Interfaces 2010, 2, 2987-2991.

(48) Lu, W.; Wang, Y.; Cao, X.; Li, L.; Dong, J.; Qian, W. Multiplexing Determination of Lung Cancer Biomarkers Using Electrochemical and Surface-Enhanced Raman Spectroscopic Techniques. New J. Chem. 2015, 39, 5420-5430.

(49) Garcia, J. C.; de Lima, D. B.; Assali, L. V. C.; Justo, J. F. Group IV Graphene- and Graphane-Like Nanosheets. J. Phys. Chem. C 2011, $115,13242-13246$.

(50) Ni, Z.; Liu, Q.; Tang, K.; Zheng, J.; Zhou, J.; Qin, R.; Gao, Z.; $\mathrm{Yu}, \mathrm{D}$.; Lu, J. Tunable Bandgap in Silicene and Germanene. Nano Lett. 2012, 12, 113-118.

(51) Tran, V.; Soklaski, R.; Liang, Y.; Yang, L. Layer-Controlled Band Gap and Anisotropic Excitons in Few-Layer Black Phosphorus. Phys. Rev. B 2014, 89, No. 235319.

(52) Novoselov, K. S.; Fal'ko, V. I.; Colombo, L.; Gellert, P. R.; Schwab, M. G.; Kim, K. A Roadmap for Graphene. Nature 2012, 490, 192-200.

(53) Song, L.; Balicas, L.; Mowbray, D. J.; Capaz, R. B.; Storr, K.; Ci, L.; Jariwala, D.; Kurth, S.; Louie, S. G.; Rubio, A.; Ajayan, P. M. Anomalous Insulator-Metal Transition in Boron Nitride-Graphene Hybrid Atomic Layers. Phys. Rev. B 2012, 86, No. 075429.

(54) Tian, J.; Li, H.; Asiri, A. M.; Al-Youbi, A. O.; Sun, X. Photoassisted Preparation of Cobalt Phosphate/Graphene Oxide Composites: A Novel Oxygen-Evolving Catalyst with High Efficiency. Small 2013, 9, 2709-2714.

(55) Mishra, A.; Mehta, A.; Basu, S.; Shetti, N. P.; Reddy, K. R.; Aminabhavi, T. M. Graphitic carbon nitride $\left(\mathrm{g}-\mathrm{C}_{3} \mathrm{~N}_{4}\right)$-based metalfree photocatalysts for water splitting: A review. Carbon 2019, 149, 693-721.

(56) Kumru, B.; Barrio, J.; Zhang, J.; Antonietti, M.; Shalom, M.; Schmidt, B. V. K. J. Robust Carbon Nitride-Based Thermoset Coatings for Surface Modification and Photochemistry. ACS Appl. Mater. Interfaces 2019, 11, 9462-9469.

(57) Rashidizadeh, A.; Ghafuri, H.; Zand, H. R. E.; Goodarzi, N. Graphitic Carbon Nitride Nanosheets Covalently Functionalized with Biocompatible Vitamin $\mathrm{B}_{1}$ : Synthesis, Characterization, and Its Superior Performance for Synthesis of Quinoxalines. ACS Omega 2019, 4, 12544-12554.
(58) Kofuji, Y.; Ohkita, S.; Shiraishi, Y.; Sakamoto, H.; Tanaka, S.; Ichikawa, S.; Hirai, T. Graphitic Carbon Nitride Doped with Biphenyl Diimide: Efficient Photocatalyst for Hydrogen Peroxide Production from Water and Molecular Oxygen by Sunlight. ACS Catal. 2016, 6, $7021-7029$.

(59) Vidyasagar, D.; Ghugal, S. G.; Umare, S. S.; Banavoth, M. Extended $\pi$-Conjugative n-p Type Homostructural Graphitic Carbon Nitride for Photodegradation and Charge-Storage Applications. Sci. Rep. 2019, 9, No. 7186.

(60) Ghafuri, H.; Jafari, G.; Rashidizadeh, A.; Manteghi, F. Co ${ }^{2+}$ Immobilized on Highly Ordered Mesoporous Graphitic Carbon Nitride (ompg- $\mathrm{C}_{3} \mathrm{~N}_{4} / \mathrm{Co}^{2+}$ ) as an Efficient and Recyclable Heterogeneous Catalyst for One-pot Tandem Selective Photo-oxidation/ Knoevenagel Condensation. Mol. Catal. 2019, 475, No. 110491. 